\title{
A collaborative care intervention improved depression outcomes, but not glycaemic control, in diabetes and comorbid depression
}

Katon WJ, Von Korff M, Lin EHB, et al. The Pathways Study: a randomized trial of collaborative care in patients with diabetes and depression. Arch Gen Psychiatry 2004;61:1042-9.

In patients with diabetes mellitus and comorbid major depression or dysthymia, does a pathways collaborative care intervention (PCCI) for depression improve both depression and glycaemic control outcomes?

METHODS

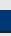

Design: randomised controlled trial.

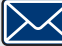

Allocation: $\{$ concealed\}.*

Blinding: blinded (outcome assessors)

Follow up period: 12 months

Setting: 9 Group Health Cooperative primary care clinics in western Washington, USA.

Patients: 329 ambulatory patients (mean age 58 y, $65 \%$ women with diabetes mellitus and comorbid major depression or dysthymia. Exclusion criteria included care from a psychiatrist, confusion suggestive of dementia, or a diagnosis of bipolar disorder or schizophrenia.

$\mathbf{R}_{\mathbf{X}}$

Intervention: a PCCI ( $n=164)$ or usual care (UC) $(n=165)$. The $\mathrm{PCCl}$ was an individualised, stepped care depression treatment programme delivered by a depression clinical specialist nurse in collaboration with a primary care physician. Components of $\mathrm{PCCl}$ included antidepressant medication, problem solving treatment, and psychiatric consultation.

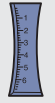

Outcomes: change in depression (20 depression items from the Hopkins Symptom Checklist-90 [SCL-90] and Patient Global Impression scores) and haemoglobin $\mathrm{A}_{1 \mathrm{c}}$ concentration measured at 3,6 , and 12 months.

II Patient follow up: $88 \%$ at 12 months.

*Information provided by author.

\section{MAIN RESULTS}

Improvement in depression (reduction in SCL-90 scores) from baseline was greater in the PCCI group than in the UC group $(p=0.004)$. More patients in the PCCI group than in the UC group had $a \geqslant 40 \%$ decrease from baseline in SCL-90 scores at 12 months (table) or improvement on Global Impression scores at $6(\mathrm{p}<0.05)$ For correspondence: $\mathrm{Dr}$ W $\mathrm{W}$ Katon, University of Washington School of Medicine, Seattle, WA, USA. wkaton@u.washington.edu

Source of funding: National Institute of Mental Health Services Division, Bethesda, MD and 12 months (table). The groups did not differ for haemoglobin $\mathrm{A}_{1 \mathrm{c}}$ concentrations throughout follow up.

\section{CONCLUSION}

In patients with diabetes mellitus and comorbid major depression or dysthymia, an enhanced process of care for depression improved depression related outcomes but not glycaemic control.

Commentary

D epression, a common comorbid condition with diabetes, has been found to be associated with hyperglycaemia. ${ }^{1}$ However, the mechanisms linking depression to glycaemic control are not well understood.' The study by Katon et al adds to our knowledge of whether interventions for depression improve both depression and glycaemic control. In 4 previous studies examining these relations, only one ${ }^{2}$ showed reductions in both glycaemic control and depressive symptoms.

The $\mathrm{PCCl}$ involved trained specialty nurses as the centrepiece of the intervention, in collaboration with a primary care physician, to deliver individualised stepped care for depression. Multifaceted interventions, especially those that include a nurse case manager and/or patient education, have shown improvements in process outcomes and patient outcomes such as glycaemic control. ${ }^{3} 2$ areas related to this collaborative intervention are noteworthy. Firstly, although the authors generally described the duration of each of the 3 steps and the percentage of patients who used the different intervention components (ie, medication, problem solving, or a combination of both), the percentages of patients who received step 1 and those who eventually progressed to step 2 and beyond were not clearly identified. Secondly, the intervention was service intensive, with the $\mathrm{PCCl}$ group being 29 times more likely to have $\geqslant 4$ additional specialty mental health visits.

The study by Katon et al adds to the body of evidence showing that enhanced depression care improves depression but not glycaemic control. These findings highlight the need for greater integration of biopsychosocial models by clinicians and researchers to advance our understanding of the important associations between depression and hyperglycaemia.

Sandra L Upchurch, RN, PhD, CDE University of Texas Houston School of Nursing Houston, Texas, USA

1 Lustman PJ, Anderson RJ, Freedland KE, et al. Depression and poor glycemic control: a meta-analytic review of the literature. Diabetes Care 2000;23:934-42.

2 Lustman PJ, Griffith LS, Freedland KE, et al. Cognitive behavior therapy for depression in type 2 diabetes mellitus. A randomized, controlled trial. Ann Intern Med 1998;129: 613-21.

3 Renders CM, Valk GD, Griffin SJ, et al. Interventions to improve the management of diabetes in primary care, outpatient, and community settings: a systematic review. Diabetes Care 2001;24:1821-33.

A pathways collaborative care intervention $(\mathrm{PCCl}) v$ usual care (UC) in patients with diabetes mellitus and comorbid major depression or dysthymia *

\begin{tabular}{llll}
\hline Outcomes at 12 months & PCCI & UC & RBI (95\% CI) \\
\hline$\geqslant 40 \%$ decrease in SCL-90 from baseline & $54 \%$ & $38 \%$ & $42 \%(10$ to 85$)$ \\
Improved patient Global Impression scores (change from baseline) & $72 \%$ & $42 \%$ & $70 \%(38$ to 113$)$
\end{tabular}

${ }^{*} \mathrm{SCL}=$ Hopkins Symptom Checklist; other abbreviations defined in glossary. RBI, NNT, and Cl calculated from data in article. 